\title{
Liquid Pipeline Transient Flow Analysis
}

\author{
Enbin Liu ${ }^{*}, 1$, Shujun Zhu ${ }^{1}$, Junying $\mathrm{Li}^{2}$, Ping Tang ${ }^{3}$, Yuhang Yang ${ }^{4}$ and Di Wang ${ }^{1}$ \\ ${ }^{1}$ Southwest Petroleum University, Chengdu, China; ${ }^{2}$ Sichuan Kehong Oil \& Gas Engineering Co., Ltd, China; ${ }^{3}$ Sichuan \\ Shida Energy Development Co., Ltd., Chengdu, China; ${ }^{4}$ Offshore Oil Engineering Co., Ltd., Tianjin, China
}

\begin{abstract}
When transient flow occurs in the liquid pipeline, it will result in higher water hammer pressure or negative pressure in pipeline, thus damaging pipeline. Through the establishment of liquid pipeline transient flow mathematical model which is solved by characteristic linear method, and by getting difference quotient equation of characteristic equation by explicit characteristic difference method, we can obtain analytical solutions of each transient flow parameters. After performing numerical simulation for pump start-up working condition, the result shows that the proposed model approach in this paper is feasible.
\end{abstract}

Keywords: Liquid pipeline, transient flow, characteristic line, simulation.

\section{INTRODUCTION}

Liquid pipelines often come across hydraulic transient due to various reasons. Hydraulic transient process often has high or low pressure, which damage pipeline and ancillary equipment when severe [1]. Therefore, it is necessary to calculate transient process which may occur during design and operation of the pipeline, in order to allow sufficient margin in design or control the effect of transient process while running. Characteristic line method [2] is the classical method for the hydraulic transient process. This paper uses central difference method to deal with transient flow characteristic equation and simulates centrifugal pump start-up working condition of liquid pipeline running. The result shows that the proposed model handling method in this paper is feasible.

\section{MATHEMATICAL MODEL OF TRANSIENT FLOW ANALYSIS}

Fluid flow in the pipe can be regarded as one dimensional flow, as fluid movement must satisfy mass conservation, momentum conservation, and energy conservation. Therefore, the model establishment of fluid movement in pipe can be based on hydrodynamics, which includes continuity equation, momentum equation and energy equation. These equations describe the relationships between pressure, temperature, and flow etc. in pipe [3, 4].

$$
\left\{\begin{array}{l}
\frac{\partial P}{\partial t}+V \frac{\partial P}{\partial x}+\rho a^{2} \frac{\partial V}{\partial x}=0 \\
\frac{\partial V}{\partial t}+\frac{1}{\rho} \frac{\partial P}{\partial x}+V \frac{\partial V}{\partial x}=-g \sin \theta-\frac{\lambda}{2 D} V|V| \\
\frac{\partial T}{\partial t}+\frac{T}{\rho C}\left(\frac{\partial P}{\partial T}\right)_{\rho} \frac{\partial V}{\partial x}+V \frac{\partial T}{\partial x}-\lambda=\lambda \frac{|V|^{3}}{2 D C}-\frac{4 K}{\rho D C}\left(T-T_{0}\right)
\end{array}\right.
$$

*Address correspondence to this author at the Southwest Petroleum University, Chengdu, China; Tel: +86-139-82069645; E-mail: Sunriselebpsb@163.com where, $\rho$-Medium density, $\mathrm{kg} / \mathrm{m}^{3} ; P$-The tube pressure, $\mathrm{Pa}$; $\theta$-The inclination between pipe and horizontal plane, rad; $t$-Time variables, $\mathrm{s} ; \quad V$-Medium flow rate, $\mathrm{m} / \mathrm{s} ; D$-Internal diameter of the pipeline, $\mathrm{m} ; g$ - Gravitational acceleration, $\mathrm{m} / \mathrm{s}^{2}$; $x$-Pipe position variables, $\mathrm{m}$; $K$-Total heat transfer coefficient of the pipeline, $\mathrm{W} /\left(\mathrm{m}^{2} . \mathrm{K}\right) ; C$-Medium heat capacity, $\mathrm{J} /(\mathrm{kg} . \mathrm{K})$; $T$-Medium temperature, $\mathrm{K} ; T_{0}$-Ground temperature, $\mathrm{K}$; $\lambda$-Pipeline hydraulic friction coefficient.

\section{MODEL SOLUTION}

The basic equations of liquid pipeline unstable flow is quasi-linear hyperbolic partial differential equations, and unstable flow belongs to fast transient flow problems. Fast transient flow can complete its transformation of flow state in short time, but during the transition process, the flow state parameters demonstrate various large change rate with time, so the shortest time step should be considered while calculation. Practice shows that explicit characteristic difference method is the better algorithm in solving transient flow, and it can ensure that calculation process has higher precision and better stability $[5,6]$.

$A=\left[\begin{array}{ccc}V & \rho a^{2} & 0 \\ \frac{1}{\rho} & V & 0 \\ 0 & \frac{T}{\rho C}\left(\frac{\partial \mathrm{P}}{\partial T}\right)_{\rho} & V\end{array}\right]$

According to linear algebra, the characteristic value $\lambda$ of A must be satisfied:

$|\lambda I-A|=0$

In this equation, $I$ is the third order unit matrix, and $|\lambda I-A|$ is the third order determinant. From the equation, we can work out three character values of $\mathrm{A}$ : 
$\left\{\begin{array}{l}\lambda_{1}=V+a \\ \lambda_{2}=V-a \\ \lambda_{3}=V\end{array}\right.$

The corresponding eigenvectors for each of $\lambda_{i}$ is:

$$
\left\{\begin{array}{l}
L^{(1)}=(1, \rho a, 0) \\
L^{(2)}=(1,-\rho a, 0) \\
L^{(3)}=\left(-\frac{T}{\rho C}\left(\frac{\partial P}{\partial T}\right)_{\rho}, 0,1\right)
\end{array}\right.
$$

Thus, according to the general form of the characteristic equations described above, we can estimate the characteristic equations of hyperbolic equations (1):

The positive characteristic line equation $\mathrm{C}^{+}$

$$
\left\{\begin{array}{l}
\frac{d x}{d t}=V+a \\
\frac{d V}{d t}+\frac{1}{\rho a} \frac{d P}{d t}=-g \sin \theta-\frac{\lambda}{2 D} V|V|
\end{array}\right.
$$

The negative characteristic line equation $\mathrm{C}^{-}$

$$
\left\{\begin{array}{l}
\frac{d x}{d t}=V-a \\
\frac{d V}{d t}-\frac{1}{\rho a} \frac{d P}{d t}=-g \sin \theta-\frac{\lambda}{2 D} V|V|
\end{array}\right.
$$

Temperature characteristic linear equation $\mathrm{V}$

$$
\left\{\begin{array}{l}
\frac{d x}{d t}=V \\
\frac{d(C T)}{d t}-\frac{T}{\rho}\left(\frac{\partial P}{\partial T}\right)_{\rho} \frac{\partial V}{\partial x}=+\lambda \frac{|V|^{3}}{2 D}-\frac{4 K}{\rho D}\left(T-T_{0}\right)
\end{array}\right.
$$

Put continuity equation into equation (8), temperature characteristic linear equation can be rewritten in the following form:

$$
\left\{\begin{array}{l}
\frac{d x}{d t}=V \\
\frac{d(C T)}{d t}-\frac{P}{\rho^{2} a^{2}} \frac{d P}{d t}=+\lambda \frac{|V|^{3}}{2 D}-\frac{4 K}{\rho D}\left(T-T_{0}\right)
\end{array}\right.
$$

The above is characteristic linear mathematical model, and for its solution we can use characteristic linear method with three different characteristic directions $\lambda_{1}=V$, $\lambda_{2}=V+a, \lambda_{3}=V-a$ to transform the partial differential equations into ordinary differential equations.

Define pipeline length as L, data collect system collect cycle as $\tau$, and divide pipeline as $n$ average parts, then use pipe step length $\Delta x$ and time step length $\Delta t$ to disperse $\mathrm{x}$ - $\mathrm{t}$ plane grid, shown as Fig. (1).

To ensure the stability of differential equation, pipe step length $\Delta x$ and time step length $\Delta t$ must satisfy with equation (10), if not it still need to be interpolatedly satisfied.
$\Delta t \leq \frac{\Delta x}{|V|+a}=\min \left(\frac{\Delta x}{|V|+a}, \frac{\Delta x}{\mid V+a}, \frac{\Delta x}{|V|}\right)$

For transient simulation of pipeline, three parameters of the first and last boundary pressure, temperature and flow of these six parameters are used generally to perform the simulation calculation. In Fig. (1), using known parameters of points(i-1,j-1)、(i,j-1)、(i+1,j-1) can obtain (i,j) point parameters.

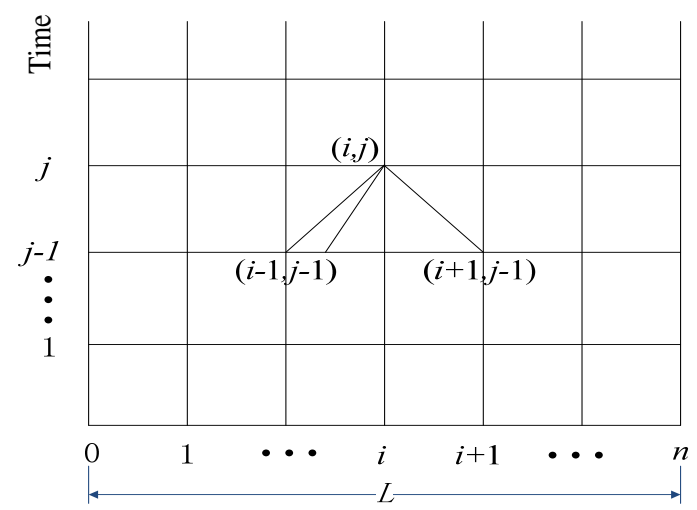

Fig. (1). Feature line mesh.

Establishment of characteristic finite difference equations is intuitive, and the equation is easy to solve. We consider that the results from explicit characteristic difference method are satisfied, mainly because the calculation steps of this method are quite small, and characteristic line is short and approximate to straight line. These results are corresponded to the hypothesis when establishing difference equation.

Central difference quotient equation of characteristic line equation can be obtained according to the equations (6), (7) and (9).

$\mathrm{C}^{+}: \frac{V_{i, j}-V_{i-1, j-1}}{\Delta t}+\frac{P_{i, j}-P_{i-1, j-1}}{\rho a \Delta t}+g \sin \theta+\frac{\lambda}{8 D}\left(V_{i, j}+V_{i-1, j-1}\right)^{2}=0$

$\mathrm{C}^{-}: \frac{V_{i+1, j-1}-V_{i, j}}{\Delta t}-\frac{P_{i+1, j-1}-P_{i, j}}{\rho a \Delta t}+g \sin \theta+\frac{\lambda}{8 D}\left(V_{i+1, j-1}+V_{i, j}\right)^{2}=0$

$\mathrm{V}: C \frac{T_{i, j}-T_{L}}{\Delta t}-\frac{P_{i, j}+P_{L}}{2 \rho^{2} a^{2}} \frac{P_{i, j}-P_{L}}{\Delta t}=\lambda \frac{\left(V_{i, j}+V_{L}\right)^{3}}{16 D}-\frac{2 K}{\rho D}\left(T_{i, j}+T_{L}-2 T_{0}\right)$

where:

$$
\begin{aligned}
& V_{L}=\frac{V_{i, j-1}}{1-\left(V_{i-1, j-1}-V_{i, j-1}\right) \frac{\Delta t}{\Delta x}} \\
& P_{L}=\left(P_{i-1, j-1}-P_{i, j-1}\right) \frac{\Delta t}{\Delta x} V_{L}+P_{i, j-1} \\
& T_{L}=\left(T_{i-1, j-1}-T_{i, j-1}\right) \frac{\Delta t}{\Delta x} V_{L}+T_{i, j-1}
\end{aligned}
$$

Combined with the boundary conditions, to simultaneous solve equations (11), (12) and (13), so that $V_{i, j}, P_{i, j}, T_{i, j}$, of next time layer can be obtained. 


$$
\begin{aligned}
V_{i, j}= & \frac{V_{i-1, j-1}+V_{i+1, j-1}-\frac{P_{i+1, j-1}-P_{i-1, j-1}}{\rho a}-\frac{\lambda \Delta t}{8 D}\left(V_{i-1, j-1}^{2}-V_{i+1, j-1}^{2}\right)}{2+\frac{\lambda \Delta t}{4 D}\left(V_{i-1, j-1}-V_{i+1, j-1}\right)} \\
P_{i, j}= & \frac{V_{i-1, j-1}-V_{i+1, j-1}}{2} \rho a+\frac{P_{i+1, j-1}+P_{i-1, j-1}}{2} \\
& -\frac{\lambda \rho \Delta t}{16 D}\left[\left(V_{i-1, j-1}+V_{i, j}\right)^{2}+\left(V_{i+1, j-1}+V_{i, j}\right)^{2}\right]-\rho g \Delta x \sin \theta \\
T_{i, j}= & \frac{\frac{P_{i, j}+P_{L}}{2 \rho^{2} a^{2}} \frac{P_{i, j}-P_{L}}{\Delta t}+\frac{\lambda\left(V_{i, j}+V_{L}\right)^{3}}{16 D}-\frac{2 K\left(T_{L}-2 T_{0}\right)}{\rho D}+\frac{T_{L} C}{\Delta t}}{\frac{C}{\Delta t}+\frac{2 K}{\rho D}}
\end{aligned}
$$

\section{CASE ANALYSIS}

Centrifugal pump pipeline or the suction pipeline's length is $1500 \mathrm{~m}$, which is connected with a constant pressure head of $20 \mathrm{~m}$ reservoir; outlet pipeline's length is $10 \mathrm{~km}$, which is connected with constant pressure head of $50 \mathrm{~m}$ liquid surface. The outside diameter of suction and outlet pipeline is $273 \mathrm{~mm}$, and wall thickness is $5 \mathrm{~mm}$. The initial steady-state flow is $0.1 \mathrm{~m}^{3} / \mathrm{s}$. We use the above method to simulate centrifugal start up process pump. The Fig. (2) shows the pressure variation regulation of pump suction port, outlet port and in the pipeline when pump starts. Fig. (3) shows the flow variation regulation of pump suction port, outlet port and in the pipeline when pump starts. From these figures we can see that after pump starts up, due to the small flow, the pump outlet pressure increases rapidly, reaching maximum $248 \mathrm{~m}$, then with a gradual increase in the flow, the pump outlet pressure gradually becomes small and finally converts to a stable value of about $156 \mathrm{~m}$. Through numerical simulation, we can master variation regulation of pipeline system parameters, such as pressure, flow and so on, and these rules provide technical support for establishing pipeline running programs.

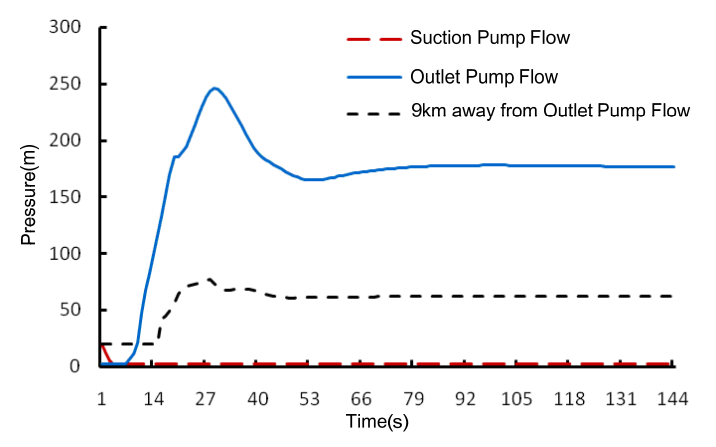

Fig. (2). The pressure fluctuation curve of different positions.

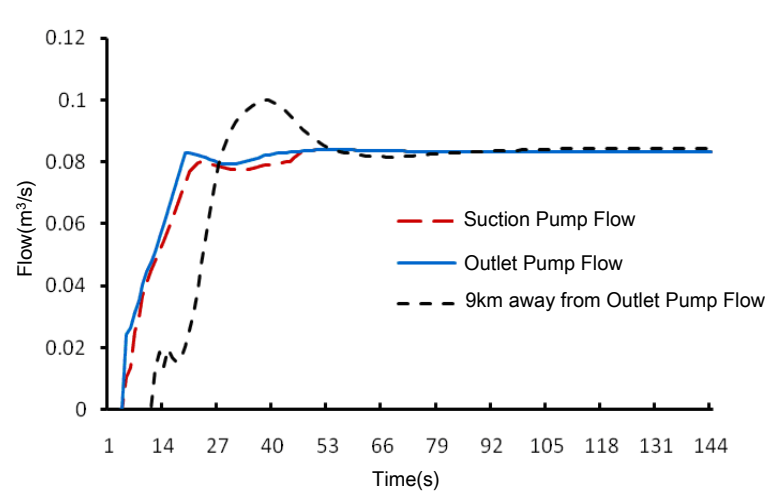

Fig. (3). The flow fluctuation curve of different positions.

\section{CONCLUSION}

Liquid pipeline often comes across hydraulic transient due to various reasons. Hydraulic transient process often generates too high or too low pressure, which will damage pipelines and ancillary equipments when serious. Therefore, it is necessary to simulate and analyse the transient process which may occur during pipe running process, in order to leave enough margin during design or take correspond measures to control effect causing by transient process when running.

\section{CONFLICT OF INTEREST}

The authors confirm that this article content has no conflict of interest.

\section{ACKNOWLEDGEMENTS}

This paper is a project supported by the special fund of China's central government for the development of local colleges and universities - the project of national first-level discipline in Oil and Gas Engineering, and the National Natural Science Foundation of China (No.51174172), and sub-project of National science and technology major project of China (No.2011ZX05054).

\section{REFERENCES}

[1] Wang, X.; Ye, H.; Tang, R. Industrial pipeline water hammer. Science Press: Beijing, 1995, pp. 78-79.

[2] Li, C.; Han, W. Unstable flow analysis of oil pipeline with gas. Oil Gas Stor Transport, 2006, 25(2), 23-27.

[3] Liu, E.; Li, C. Research and application of the technology of pipeline leak detection. Oil Gas Stor Transport, 2006, 28(5), 43-48.

[4] Shashi, M. Liquid pipeline hydraulics. SYSTEK Technol. Inc: USA, 2004, pp. 133-136.

[5] Liu, Z.; Liu, G. Water hammer and protection of pump station. Beijing: Water Conservancy and Electric Power Press, 1985, 102105 .

[6] Bruce, EL.; Roland, W. J. Hydraulics of Pipeline Systems. CRC Press: USA, 2000, pp. 87-91. 